\title{
Analysis on the black hole formations inside old neutron stars by isospin-violating dark matter with self-interaction
}

\author{
Yen-Hsun Lin ${ }^{a, *}$ and Guey-Lin Lin ${ }^{b}$ \\ ${ }^{a}$ Institute of Physics, Academia Sinica, \\ No. 128, Sec. 2 Academia Rd., Taipei 115, Taiwan \\ ${ }^{b}$ Institute of Physics, National Chiao Tung University \\ No. 1001, University Rd., Hsinchu 300, Taiwan \\ E-mail: yenhsun@gate.sinica.edu.tw, gueylin.lin@gmail.com
}

Fermionic dark matter (DM) with attractive self-interaction is possible to form black holes (BH) inside the Gyr-old neutron stars (NS). Therefore by observing such NS corresponding to their adjacent DM environments can place bounds on DM properties, eg. DM-baryon cross section $\sigma_{\chi}$, DM mass $m_{\chi}$, dark coupling $\alpha_{\chi}$ and mediator mass $m_{\phi}$. In case of isospin violation, DM couples to neutron and proton in different strengths. Even NS is composed of protons roughly one to two percent of the total baryons, the contribution from protons to the DM capture rate could be drastically changed in the presence of isospin violation. We demonstrate that this effect can be important in certain cases. On the other hand, DM-forming BH inside the star is subject to many criteria and the underlying dynamics is rich with interesting features. We also systematically review the relevant physics based on the virial equation.

40th International Conference on High Energy Physics - ICHEP2020

Prague, Czech Republic (virtual meeting)

July 28 - August 6, 2020

\footnotetext{
${ }^{*}$ Speaker
} 


\section{Introduction}

Dark matter (DM) composites one-fifth of the Universe but its particle essence remains elusive. To discern the nature of DM is a great challenge in modern physics. Plethora of experiments to detect the signal from dark sector either from the direct interaction between DM and the Standard Model (SM) particles [1, 2] or from the indirect measurement of the events produced by DM annihilation or decay $[3,4]$ are undergoing.

If no anti-DM exists, the asymmetric DM (ADM) [5], No annihilation can happen and DM can be captured by the stellar objects, e.g. neutron stars (NSs), without depletion. The total amount of DMs grows unlimitedly until gravitational collapse is triggered and become a black hole (BH) that consumes the entire NS. A giga-year-old (Gyr-old) NS has enough time to accumulate sufficient DMs that collapse into a BH. We examine such scenario in the presence of attractive DM self-interaction. In addition, isospin symmetry is not necessary for DM-baryon interaction. The implication is discussed as well.

Without loss of generality, We assume DM self-interaction is described by the Yukawa potential

$$
V(r)=-\frac{\alpha_{\chi}}{r} e^{-m_{\phi} r}
$$

where $\alpha_{\chi}$ and $m_{\phi}$ are the dark fine structure constant and mediator mass respectively. For DMbaryon interaction, we distinguish neutron coupling $\varepsilon_{n}$ and proton coupling $\varepsilon_{p}$ to DM, where they are given by

$$
\begin{aligned}
& \varepsilon_{n}=-\frac{\varepsilon_{Z}}{4 s_{W} c_{W}} \approx-0.6 \varepsilon_{Z}, \\
& \varepsilon_{p}=\varepsilon_{\gamma}+\frac{\varepsilon_{Z}}{4 s_{W} c_{W}}\left(1-4 s_{W}^{2}\right) \approx \varepsilon_{\gamma}+0.05 \varepsilon_{Z} .
\end{aligned}
$$

The couplings $\varepsilon_{\gamma}$ and $\varepsilon_{Z}$ arise from kinetic mixing and $Z$ mass mixing. Therefore, we can link DM-proton scattering cross section $\sigma_{\chi p}$ and DM-neutron scattering cross section $\sigma_{\chi^{n}}$ by

$$
\sigma_{\chi p}=\left(\frac{\varepsilon_{n}}{\varepsilon_{p}}\right)^{-2} \sigma_{\chi n}
$$

where $\varepsilon_{n} / \varepsilon_{p}$ is the isospin factor. If it is not unity, then isospin is violated. The phenomenological detail is left in Ref. [6].

\section{Dark matter capture and black hole formation}

\subsection{The capture mechanism}

When the halo DM particles scatter with NS and lose significant amount of energies, they are gravitationally trapped in the star. The evolution of DM number $N_{\chi}$ in NS can be characterized by the differential equation

$$
\frac{d N_{\chi}(t)}{d t}=C_{c}-C_{e} N_{\chi}(t)
$$

where $C_{c}$ is the NS capture rate due to DM-baryon scattering and $C_{e}$ is the evaporation rate. It is argued that $C_{e}$ is negligible unless $m_{\chi} \lesssim O(\mathrm{keV})$ [7]. In our interested parameter space, $C_{e}$ can be ignored so that $N_{\chi}(t)$ is given by

$$
N_{\chi}(t)=C_{c} t
$$


Thus, DM would accumulate without limit in the star. The DM self-capture is not important in this study and can be safely ignored.

The capture rate is investigated extensively in Ref. [7] and references therein. It is written as

$$
C_{c}=\int_{0}^{R_{0}} 4 \pi r^{2} d r \int_{0}^{\infty}\left(\frac{\rho_{\chi}}{m_{\chi}}\right) \frac{f(u)}{u} w(r) d u \int_{0}^{v_{\mathrm{esc}}(r)} R^{-}(w \rightarrow v) d v
$$

where $\rho_{\chi}$ is the local DM density near the NS, $f(u)$ the DM velocity distribution in the NS rest frame and assumed to be Maxwell-Boltzmannian, $v_{\mathrm{esc}}(r)$ the escape velocity at layer $r$ of the NS and $w=\sqrt{u^{2}+v_{\mathrm{esc}}^{2}(r)}$ the velocity of DM falling into layer $r$. The quantity $R^{-}(w \rightarrow v)$ is the DM differential scattering rate from the initial velocity $w$ to a smaller final velocity $v$. We also include Pauli blocking effect in $R^{-}(w \rightarrow v)$ where it suppresses the capture rate if the momentum transfer during the collision is not large enough to overcome the Fermi degeneracy pressure.

\subsection{Gravitational collapse of DM in the NS}

When DMs are captured, they will thermalize with the surrounding nucleons within a short time interval and form a dark spherical cloud. The thermal radius of the cloud $r$ can be determined by the virial equation [8]

$$
2\left\langle E_{k}\right\rangle=\frac{4}{3} \pi G \rho_{b} m_{\chi} r^{2}+\frac{G N_{\chi} m_{\chi}^{2}}{r}+\sum_{j}^{N_{\chi}-1}\left(\frac{\alpha_{\chi}}{r_{j}} e^{-m_{\phi} r_{j}}+\alpha_{\chi} m_{\phi} e^{-m_{\phi} r_{j}}\right)
$$

where $\rho_{b}$ is the NS baryon density. The first two terms on the RHS arise from gravitational potentials of NS and DM, respectively, and the last term is from the Yukawa interactions between DMs.

When NS keeps capturing DMs, $N_{\chi}$ becomes larger and DMs are degenerate when $N_{\chi} \gtrsim 10^{36}$. The corresponding Fermi energy is

$$
E_{k, \mathrm{deg}}=\frac{\left(9 \pi N_{\chi} / 4\right)^{2 / 3}}{2 m_{\chi} r^{2}} .
$$

Eventually, when too much DMs are accumulated in the star, they trigger the gravitational instability. Unless the Fermi degeneracy pressure is strong enough to overcome both the gravitational contraction and attractive DM self-interaction. DM collapse into a $\mathrm{BH}$ is inevitable. To avoid BH evaporation, it is also required that DM should collapse under

$$
N_{\chi}>3.4 \times 10^{36}\left(\frac{m_{\chi}}{\mathrm{GeV}}\right) .
$$

The mathematical detail in provided in Refs. [6, 8].

\section{Isospin violation and its implication}

In the presence of isospin violation, $\sigma_{\chi p}$ can be much larger than $\sigma_{\chi n}$. Though proton yield $n_{p}$ is roughly 50 times smaller than the neutron yield $n_{n}$ in the NS, the contribution from proton to the capture rate can be comparable or even larger than the neutron contribution. Thus, proton is taken into consideration in this work. 

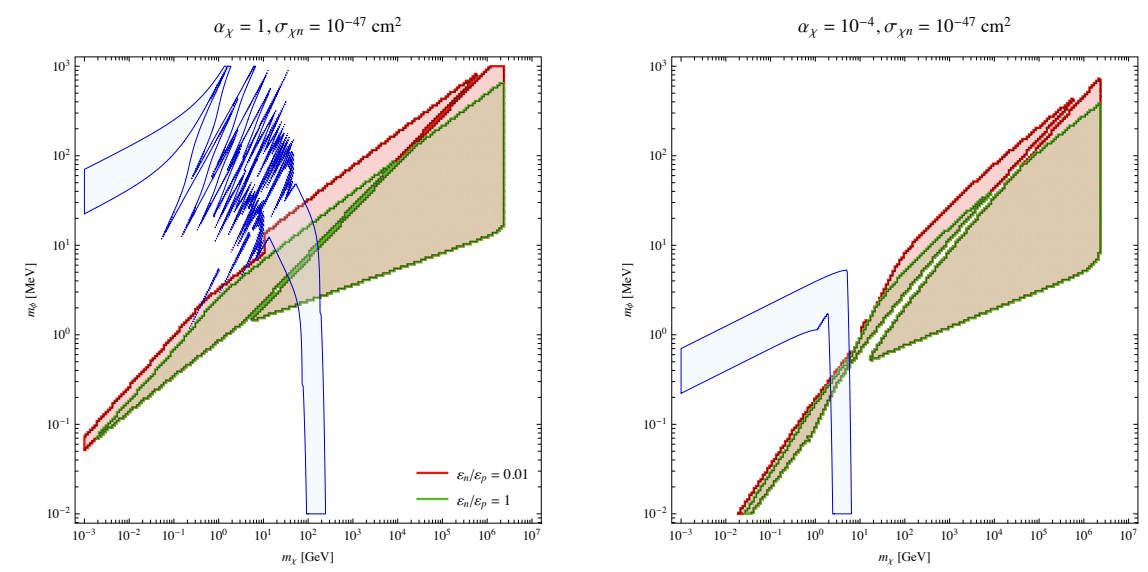

Figure 1: The exclusion plane over $m_{\chi}-m_{\phi}$ plane with $\alpha_{\chi}=1$ (left) and $10^{-4}$ (right) and $\sigma_{\chi^{n}}=10^{-47} \mathrm{~cm}^{2}$. Red shaded region is excluded by a 5-Gyr-old NS with $\varepsilon_{n} / \varepsilon_{p}=0.01$ and green with $\varepsilon_{n} / \varepsilon_{p}=1$. The DM environment has the local density $\rho_{\chi}=0.3 \mathrm{GeV} \mathrm{cm}^{-3}$ and the velocity dispersion $\bar{v}=220 \mathrm{~km} \mathrm{~s}^{-1}$. Light blue shaded region is allowed by the SIDM constraint.

Once NS captures enough DMs, it will be destroyed by the DM-collapsed BH. For a Gyr-old NS, it is capable of capturing sufficient $N_{\chi}$ to trigger this effect. The benchmark NS age is 5 Gyrs and the local DM density $\rho_{\chi}=0.3 \mathrm{GeV} / \mathrm{cm}^{3}$ in our analysis. The condition is similar to the ambience of the Solar system.

The NS sensitivity on $m_{\chi}$ over $m_{\phi}$ plane for the given $\alpha_{\chi}$ and $\sigma_{\chi n}$ is plotted in Fig. 1. The color shaded regions indicate the NS should be destroyed after 5 Gyrs. Once future observation find any NS older than the benchmark age, the associated parameter space is excluded. Green and red colors stand for different isospin violation values. The red case implies $\sigma_{\chi p}$ is 10000 times larger than $\sigma_{\chi^{n}}$. Even $n_{p} \approx n_{b} / 50$, the capture rate $C_{c}$ can still be strongly enhanced by the larger $\sigma_{\chi p}$. A sharp vertical cut to the right of each figure when $m_{\chi} \approx 10^{6} \mathrm{GeV}$, it is because the number of DM at the the collapse will never satisfy Eq. (9). Thus, BH will evaporate after it was born and NS cannot be destroyed. The light blue shaded region is the derived SIDM constraint based on the method in Ref. [9].

In addition, we also plot the NS sensitivity on $\sigma_{\chi n}$ with given $\alpha_{\chi}$ and $m_{\phi}$ in Fig. 2. The results with various isospin violation values are shown as well. The blue shaded regions are XENON1T constraints. Green shaded region indicates DM cannot thermalize with NS in 5 Gyrs, thus it renders the $E_{k}$ in the non-degenerate case cannot be determined by the NS core temperature. See Ref. [8] for detail.

\section{Summary}

In this proceeding, we have pointed out that isospin violation could significantly change the NS sensitivities to $\sigma_{\chi n}$. Up to recent NS studies, proton contribution to the capture rate is ignored due to the assumption of isospin symmetry in the fermionic DM-baryon interaction. On the other hand, we have demonstrated that the proton contribution to the capture rate becomes comparable and even exceeds the neutron contribution for $\varepsilon_{n} / \varepsilon_{p}$ much smaller than unity. Thus the sensitivity regions are enlarged. This has not been taken into account for the case of attractive fermionic DM. In our 

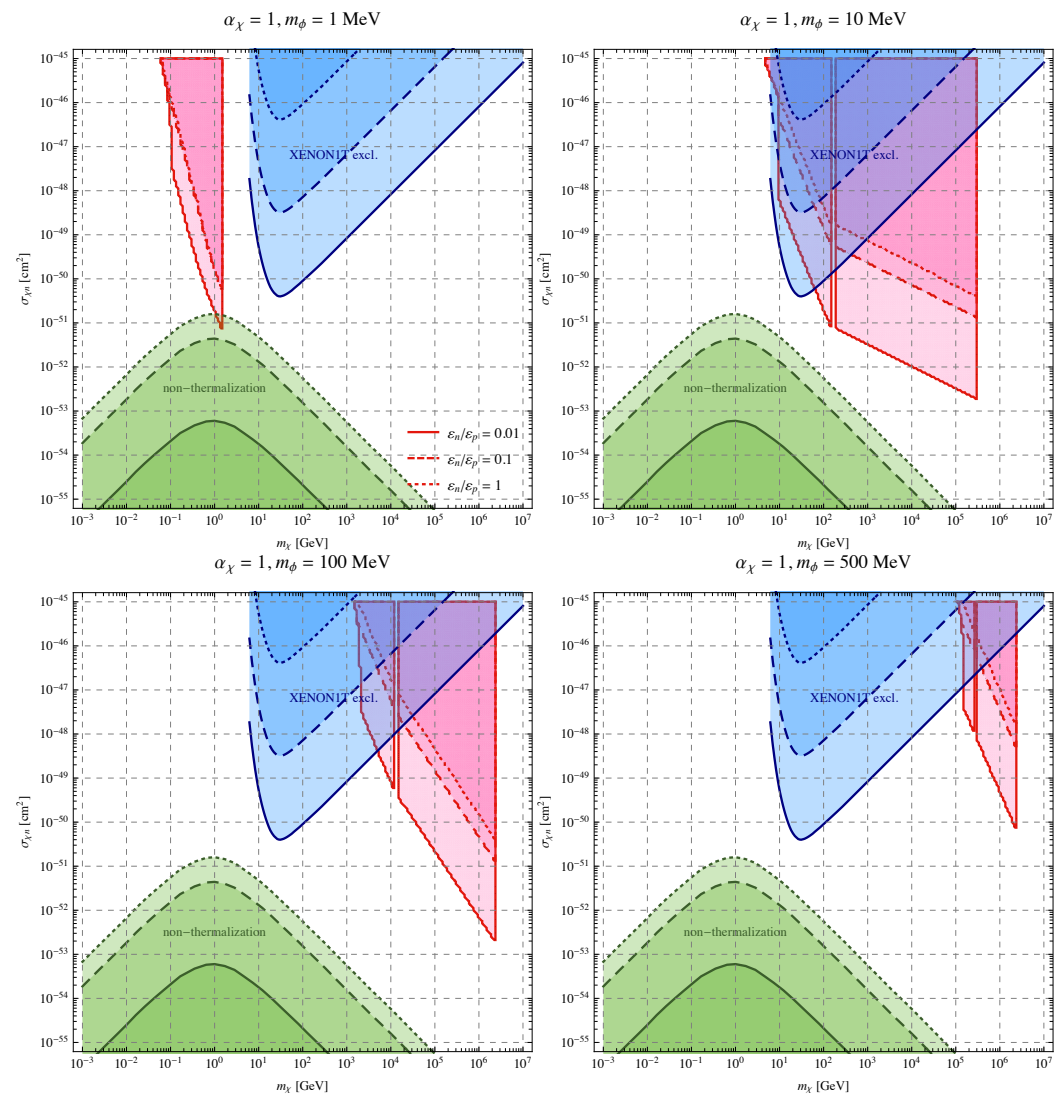

Figure 2: The NS sensitivities on $\sigma_{\chi^{n}}$ with $\alpha_{\chi}=1$. The solid, dashed and dotted lines correspond to $\varepsilon_{n} / \varepsilon_{p}=0.01,0.1$ and 1 , respectively. Pink-shaded regions are excluded by a 5-Gyr-old NS. Blue-shaded regions are XENON1T exclusions and green-shaded region indicates the parameter range that DM cannot thermalize with NS within 5 Gyrs.

new study, the DM capture rate is based on a more realistic NS profile. Moreover, the suppression due to Pauli blocking effect has been considered in calculating $C_{c}$.

For the DM-forming BH inside the NS, the fermionic case contains very rich dynamics as we have summarized in Sec. 2. A systematic algorithm to compute the fate of NS based on the DM particle parameters $\alpha_{\chi}, m_{\chi}, m_{\phi}$ and the captured $N_{\chi}$ in the NS is given in Ref. [8].

In closing, we stress that the microscopic feature of DM self-interaction is not yet well understood at the present time. Thus, if DM self-interaction is repulsive instead of attractive, the last term in the virial equation, Eq. (7), changes sign and consequently counteracts the gravitational attraction. This effect would disfavor the collapse of DMs into a BH. Hence NS is no longer able to constrain DM parameter space. On the other hand, if DMs attract each other as studied in this work, it is possible for them to form a large nugget that contains enormous number of DM particles as investigated in Refs. [10,11]. The nugget may have different scattering cross section with the baryons in NS. Hence the method employed here for constraining DM properties should be modified. We relegate this to future studies. 


\section{References}

[1] XENON collaboration, First Dark Matter Search Results from the XENONIT Experiment, Phys. Rev. Lett. 119 (2017) 181301 [1705. 06655].

[2] XENON collaboration, Dark Matter Search Results from a One Ton-Year Exposure of XENONIT, Phys. Rev. Lett. 121 (2018) 111302 [1805.12562].

[3] Fermi-LAT collaboration, The Fermi Galactic Center GeV Excess and Implications for Dark Matter, Astrophys. J. 840 (2017) 43 [1704. 03910].

[4] DAMPE collaboration, Direct detection of a break in the teraelectronvolt cosmic-ray spectrum of electrons and positrons, Nature 552 (2017) 63 [1711. 10981].

[5] K. Petraki and R.R. Volkas, Review of asymmetric dark matter, Int. J. Mod. Phys. A 28 (2013) 1330028 [1305.4939].

[6] G.-L. Lin and Y.-H. Lin, Analysis on the black hole formations inside old neutron stars by isospin-violating dark matter with self-interaction, JCAP 08 (2020) 022 [2004 . 05312].

[7] R. Garani, Y. Genolini and T. Hambye, New Analysis of Neutron Star Constraints on Asymmetric Dark Matter, JCAP 05 (2019) 035 [1812.08773].

[8] J. Bramante, K. Fukushima, J. Kumar and E. Stopnitzky, Bounds on self-interacting fermion dark matter from observations of old neutron stars, Phys. Rev. D 89 (2014) 015010 [1310.3509].

[9] S. Tulin, H.-B. Yu and K.M. Zurek, Beyond Collisionless Dark Matter: Particle Physics Dynamics for Dark Matter Halo Structure, Phys. Rev. D 87 (2013) 115007 [1302 . 3898].

[10] M.B. Wise and Y. Zhang, Stable Bound States of Asymmetric Dark Matter, Phys. Rev. D 90 (2014) 055030 [1407.4121].

[11] M.I. Gresham, H.K. Lou and K.M. Zurek, Nuclear Structure of Bound States of Asymmetric Dark Matter, Phys. Rev. D 96 (2017) 096012 [1707.02313]. 